\title{
Totally correct logic program transformations via well-founded annotations
}

\author{
Alberto Pettorossi • Maurizio Proietti
}

Published online: 23 May 2008

(C) Springer Science+Business Media, LLC 2008

\section{Erratum to: Higher-Order Symb Comput \\ DOI 10.1007/s10990-008-9024-6}

The original version of this article unfortunately contained a mistake. The text of Theorem 4 was incorrect. The corrected theorem is given below.

Theorem 4 The set $\mathcal{P}\left(B_{\mathcal{W}}\right)$ is a complete lattice with respect to set inclusion and, for every annotated program $\bar{P}$, the immediate consequence operator $T_{\bar{P}}: \mathcal{P}\left(B_{\mathcal{W}}\right) \rightarrow \mathcal{P}\left(B_{\mathcal{W}}\right)$ is a continuous function. Thus, $T_{\bar{P}}$ has a least fixpoint $\operatorname{lfp}\left(T_{\bar{P}}\right)$ and a greatest fixpoint $g f p\left(T_{\bar{P}}\right)$. Moreover, $\operatorname{lfp}\left(T_{\bar{P}}\right)$ is the least upper bound of the chain $\left\{T_{\bar{P}}^{n}(\emptyset) \mid n \in \mathbb{N}\right\}$ and $\operatorname{lfp}\left(T_{\bar{P}}\right)$ is the least $\mathcal{W}$-model $M(\bar{P})$ of $\bar{P}$.

The online version of the original article can be found under doi: 10.1007/s10990-008-9024-6.

\footnotetext{
A. Pettorossi $(\bowtie)$

e-mail: pettorossi@disp.uniroma2.it

M. Proietti

IASI-CNR, Viale Manzoni 30, Roma, Italy

e-mail: proietti@iasi.cnr.it
}

DISP, University of Roma Tor Vergata, Via del Politecnico 1, 00133 Roma, Italy 